\title{
Accelerating and Increasing Nano-scaled Pores Formation on Electrospun Poly(3-hydroxybutyrate-co-3-hydroxyvalerate) Fibers
}

\author{
Lanxin Lyu ${ }^{12}$, Ningping Huang ${ }^{3 *}$, and Ying Yang ${ }^{1 *}$ \\ ${ }^{1}$ Institute for Science \& Technology in Medicine, Keele University, Stoke-on-Trent, ST4 7QB, \\ United Kingdom \\ ${ }^{2}$ First Aid and Relief Medical Department, Xuzhou Medical College, Emergency Center, the \\ Affiliated Hospital of Xuzhou Medical College, Xuzhou 221006, P. R. China
}

Email:ㄴlanxin@xzmc.edu.cn

${ }^{1}$ *Corresponding author, Institute for Science \& Technology in Medicine, Keele University,

Stoke-on-Trent, ST4 7QB, United Kingdom

Email: y.yang@keele.ac.uk

${ }^{3}$ *Co-corresponding author, State Key Laboratory of Bioelectronics, School of Biological

Science and Medical Engineering, Southeast University, Nanjing 210096, P. R. China

Email: $\underline{n p h u a n g @ s e u . e d u . c n}$ 


\begin{abstract}
Porous fibers are advantageous for filtration systems, drug delivery systems and in the field of tissue engineering, in comparison to their non-porous counterparts. In this study, we developed a facile technique including two steps to generate poly(3-hydroxybutyrate-co-3- hydroxyvalerate) (PHBV) porous fibers with a controllable pore size. An electrospinning technique was employed to obtain five types of PHBV/poly(ethylene oxide) (PEO) blended fibers (PHBV:PEO = 9:1, 8:2, 7:3, 6:4, 5:5) with PEO as the porogen. PEO was leached out by simulated body fluid (SBF) and water respectively. The pore morphology and calcium deposition of the resulting fibers was compared to those formed on film through SEM-EDX analysis. It was revealed that pore size and number increased with increasing PEO percentage in the fiber or film. The pore size on the films (at micrometer scale) was much larger than that of nanofibers, which was in the range of $70-120 \mathrm{~nm}$. The simultaneous removal of PEO and deposition of calcium phosphate through SBF buffer enhanced synergistically both pore formation and mineral deposition. The different phase separation mechanism explains the different pore morphology in the film and the nanofibers. The cellular experimental results show that fibers with nanometer-scale pores and minerals can enhance the proliferation of bone marrow-derived mesenchymal stem cells.
\end{abstract}

Keywords: PHBV, porous fibers, phase separation, mineralization 


\section{Introduction}

Poly(3-hydroxybutyrate-co-3-hydroxyvalerate) (PHBV) is the copolymer of poly(hydroxybutyrate) (PHB) and poly(hydroxyvalerate) (PHV) with biodegradable, non-antigenic and biocompatible properties. It has increased in interest over recent years and is now widely used in drug delivery systems, bone tissue engineering, nerve defection repair and wound repair [1-4]. However, there are still challenges to be overcome with its use, such as slow degradation and a hydrophobic surface. PHBV has been constructed into different types of scaffolds for various applications, such as casting films, cylinders, 3-dimensinonal porous scaffolds and electrospun fibers [5-8]. As is widely acknowledged, the technique of electrospinning can generate fibrous scaffolds with fiber diameters ranging from a few micrometers to hundreds of nanometers, with a high surface area to volume ratio; an attribute comparable to the fibrous structure of the natural extracellular matrix $[9,10]$. Due to these advantages, electrospun fibers have been used in the field of tissue engineering, the filtration industry and in drug delivery systems $[11,12]$. However, the surface of fibers fabricated via electrospinning is typically pore-less. Electrospun fibers with porous surfaces are considered more advantageous for a variety of applications. For example, pore structure can enhance the anchoring points for cells, which consequently affects cell proliferation and differentiation. Furthermore, the high surface area can improve the efficiency in filtration systems and catalysis [13].

Numerous strategies have been attempted to generate porous electrospun fibers. Regulation of electrospinning parameters and use of specific solvents are the most common techniques. Michael et al. successfully obtained poly-L-lactide (PLLA), polycarbonate (PC) and polyvinylcarbazole porous fibers with a pore size around $100 \mathrm{~nm}$ through phase separation during electrospinning, by choosing the 
appropriate spinning parameters and solvent [14]. However, altering the solvent system in electrospinning can dramatically affect fiber diameter and subsequently affect the functionality. Changing humidity is another method of porous fiber manufacturing via electrospinning. It has been reported that for certain solvent systems, the evaporation of the solvent will cool the jet, leading to small, condensed water droplets on the fibers formed, becoming a porogen for pore formation. Cheryl et al. have generated polystyrene (PS) porous fibers using this method and found that increasing humidity caused an increase in the number, diameter, shape and distribution of the pores [15]. Immersing the collector in a bath of liquid nitrogen, followed by removal of the solvent in vacuum, is another smart strategy to yield porous electrospun fibers through thermally-induced phase separation, between the solvent-rich and solvent-poor regions [16]. However, porous fibers produced by this method had a larger diameter than with standard procedures, because the collection distance had to be shortened to inhibit the solvent evaporating totally before reaching the collector.

The technique combining phase separation and porogen leaching is the most commonly used method to generate porous fibers in electrospinning. In general, a second component acting as a porogen (such as inorganic particles or hydro soluble polymers) which can be easily separated and removed from the main component, is blended to yield electrospun fibers. The second component is then removed by a solvent that cannot dissolve the main component, to obtain porous fibers. Mona et al. fabricated polyacrylonitrile (PAN) porous nanofibers through a porogen leaching method. In their study, calcium carbonate $\left(\mathrm{CaCO}_{3}\right)$ particles were mixed with PAN/DMF (dimethylformamide) solution to electrospin nanofibers, and hydrochloric acid $(\mathrm{HCl})$ was used to extract $\mathrm{CaCO}_{3}$ particles [17]. Besides inorganic particles, the water soluble polymers such as gelatin, PEO and poly(vinyl-pyrrolidone) (PVP) are the 
most widely used porogens to generate porous fibers in electrospinning $[9,18,19]$. Using the same technique, Muhamad et al. successfully obtained poly(vinylidenefluoride) (PVDF) porous electrospun fibers by removing hydro soluble PVP [19].

Poly(ethylene oxide) (PEO), as one kind of hydrophilic polyester, is widely used in drug delivery systems and blood contact devices due to its low toxicity and non-immunogenicity, as well as its ability to minimize protein adsorption to surfaces [20]. PEO has also been used to improve the physical property of PHBV. In our previous research, we have employed PEO as an additive to improve continuity and uniformity of PHBV electrospun fibers [8]. Alessandra et al. have systematically studied the PHBV/PEO electrospun fiber's thermal and mechanical properties [21]. Most previous research about PHBV/PEO focused on building up a co-composite system to improve PHBV's properties. Several research groups have studied the PEO leaching technique for generating porous structured scaffolds, while there has been only few report which conducted mineralization along with pore formation.

In this study, we aim to produce PHBV porous fibers by the method of electrospinning and porogen leaching. The pore formation mechanisms will then be investigated. To achieve this, different percentages of PEO were blended into PHBV electrospun solution to fabricate as-spun PHBV/PEO fibers, which were then leached by SBF solution and water to obtain porous fibers. Fiber porosity, pore size distribution and calcium deposits were evaluated using scanning electron microscopy (SEM). At the same time, PHBV/PEO casting films (with the same concentration and components as the fibers) were prepared, to investigate the phase separation mechanism by comparing the pore formation on nanofibers against casting film. Finally, bone marrow derived mesenchymal stem cells (MSCs) were used to study 
the effect of PEO ratio and the porous surface of electrospun fibers on stem cell viability and morphology.

\section{Materials and Methods}

\subsection{PHBV/PEO electrospun fibers}

In order to produce PHBV/PEO composite fibers, electrospinning technology was employed. The procedures are thoroughly described in our previous publication [8]. Briefly, 2\% (wt/v) PHBV (12\% of HV, Sigma, USA) and PEO (1000 kD, Guoren Yikang, Beijing, China) blend solutions of different weight ratios (PHBV:PEO $=9: 1,8: 2,7: 3,6: 4,5: 5$ ) were prepared by dissolving in 2, 2, 2-trifluoroethanol (TFE, Darui, Shanghai, China). Subsequently, the solution was fed into a glass syringe with \#6 needle (inside diameter: $0.5 \mathrm{~mm}$ ) and continually driven by an advancing pump at the speed of $5 \mathrm{~mL}$ per hour. A $12 \mathrm{kV}$ DC voltage was applied between the metal needle and collector at a distance of $25 \mathrm{~cm}$. PHBV/PEO fiber samples used for cell culture work were round shaped meshes with diameters of $10 \mathrm{~mm}$.

\subsection{PHBV/PEO casting films}

Films of blends with the same ratios as listed above were obtained by casting in glass petri dishes overnight. In brief, 2\% (wt/v) PHBV and PEO blend solutions of different weight ratios (PHBV:PEO = 9:1, 8:2, 7:3, 6:4, 5:5) were made by dissolving in chloroform $\left(\mathrm{CHCl}_{3}\right.$, Sigma, UK) and casting in glass petri dishes overnight to allow the solvent to evaporate thoroughly. The flat and thin films were detached from the bottom of petri dishes gently with forceps. 


\subsection{PEO leaching and mineralization of fibers and film}

Five types of fibers and films with different PHBV and PEO ratios as stated above were immersed in simulated body fluid (SBF) solution and incubated at $37^{\circ} \mathrm{C}$ for 4 weeks. SBF solution was prepared according to a previous study [22]. In brief, $\mathrm{NaCl}(7.996 \mathrm{~g}), \mathrm{NaHCO}_{3}(0.350 \mathrm{~g}), \mathrm{KCl}(0.224 \mathrm{~g})$, $\mathrm{K}_{2} \mathrm{HPO}_{4} \bullet 3 \mathrm{H}_{2} \mathrm{O}(0.228 \mathrm{~g}), \mathrm{MgCl}_{2} \bullet 6 \mathrm{H}_{2} \mathrm{O}(0.305 \mathrm{~g}), 1 \mathrm{M} \mathrm{HCl}(40 \mathrm{~mL}), \mathrm{CaCl}_{2}(0.278 \mathrm{~g}), \mathrm{Na}_{2} \mathrm{SO}_{4}(0.071 \mathrm{~g})$ and Tris $(6.057 \mathrm{~g})$ (all purchased from Sigma, UK) was added to 1L distilled water one by one in serial order, after each reagent was completely dissolved. Using $1 \mathrm{M} \mathrm{HCl}$ the solution $\mathrm{pH}$ was adjusted to 7.4. In order to investigate the different mechanisms of pore formation on electrospun fibers, distilled water $\left(\mathrm{dH}_{2} \mathrm{O}\right)$ was also used as a leaching solution. SBF solution and $\mathrm{dH}_{2} \mathrm{O}$ were changed every 3 days for each sample. After 2 or 4 weeks of soaking, fibrous samples were taken out and gently washed three times with $\mathrm{dH}_{2} \mathrm{O}$ and then transferred into a vacuum oven overnight at $60^{\circ} \mathrm{C}$ to evaporate the residual water. Finally, the samples were weighed and stored in a desiccator for morphology analysis. The film samples were taken out of the SBF after 4 weeks, gently washed twice and dried at room temperature before further analysis.

\subsection{Fibers and film morphology analysis}

The morphology of PHBV/PEO fibers before (as-spun fibers) and after (porous fibers) being immersed in SBF solution and $\mathrm{dH}_{2} \mathrm{O}$ was observed using SEM (ultra plus Zeiss, Germany). The diameters of PHBV as-spun fibers and porous fibers were measured from SEM images based on 20 fibers per sample, using ImageJ software. The pores' percentages of PHBV porous fibers (30\%, 40\%, 50\% PEO) were calculated from SEM images by measuring the pore area of ten fibers chosen randomly using ImageJ. Pore size and distribution of these three kinds of porous fibers were calculated from SEM images by 
measuring diameters of more than 100 pores per sample using ImageJ. In order to observe the morphology of PHBV films before and after immersion in SBF, bright field inverted microscopy (Olympus, UK) was employed.

\subsection{SEM-EDX analysis}

SEM combined with energy dispersive X-ray analysis (SEM-EDX, TM3000, Hitachi, Japan) was used to analyze the mineral deposition on the surface of PHBV fibers and films after incubating in SBF for 4 weeks. The element distribution of calcium on PHBV fibers and films was quantified by scanning two different rectangle areas (length $\times$ width: $320 \mu \mathrm{m} \times 240 \mu \mathrm{m}$ ). To investigate the porous fibers effect on mineralization, PHBV porous fibers (with PEO ratios of 30\%, 40\%, and 50\%) and PHBV non-porous fibers (with PEO ratio of 10\%) were examined. To compare the effect of micropores and nanopores on the mineralization, PHBV films with the same PEO ratios were also tested.

\subsection{Cellular experiment}

The effect of PEO and porosity of PHBV fibers on cell proliferation was assessed using MSCs harvested from rat bone marrow. To evaluate the effect of PEO content on cell proliferation, five types of as-spun PHBV fibers with different ratios of PEO (9:1, 8:2, 7:3, 6:4, and 5:5) were immersed in 70\% ethanol for 1 hour to sterilize and then rinsed three times with sterile PBS before seeding cells. To evaluate the porous effect on cell proliferation and morphology, PHBV as-spun and porous fibers with $40 \%$ of PEO were prepared, and PHBV as-spun fibers with $10 \%$ PEO with smooth surface were employed as a control. 
MSCs were seeded onto the surface of fibers with a density of $2 \times 10^{4}$ cells $/ \mathrm{mL}$. After 1,3 and 5 days of seeding, cell count kit-8 (CCK-8, CCK-8, Dojindo, Japan) was used to test cell proliferation on different surfaces. Briefly, CCK-8 solutions at a dilution of 1:10 with media were added to each sample and cultured in an incubator at $37{ }^{\circ} \mathrm{C}$ with $5 \% \mathrm{CO}_{2}$. Three hours later, $100 \mu \mathrm{L}$ of media was transferred to each well of a 96-well plate to measure absorption value at a wavelength of 450nm using a microplate reader (Symergy HT, BioTek, USA). The rest of the media with CCK-8 solution was discarded and fresh culture media was added. The experiment was repeated three times with six parallel repeats for each sample.

Five days after seeding, SEM was used to evaluate cell morphology on the surfaces of as-spun and porous PHBV fibrous scaffolds with 40\% PEO and as-spun PHBV fiber scaffolds with 10\% PEO. MSCs on different surfaces were gently rinsed three times with PBS buffer and fixed with $2.5 \%$ glutaraldehyde (Sigma-Aldrich, USA) for 2 hours at room temperature. After fixation, the samples were rinsed again with PBS and dehydrated with gradient ethanol $(30 \%, 50 \%, 70 \%, 80 \%, 90 \%, 95 \%$ and $100 \%)$ for 10 minutes each step. The samples were then dried and examined by SEM.

\subsection{Statistical analysis}

For the weight measuring, 3 samples were used for each group, and for the cell culture, 6 repeats were used for each group. Results are presented as mean \pm standard deviation. Each result was statistically analyzed by t-testing. A p value of $p<0.05$ was considered statistically significant.

\section{Results}




\subsection{Characterization of as-spun and porous PHBV/PEO fibers}

3.1.1. Diameters of PHBV fibers. Figure 1(a1-e1) shows the SEM images of PHBV as-spun fibers with different ratios of PEO, from which we found that the PEO ratio affected the PHBV fiber diameter. The PHBV fiber diameter increased along with the increase in PEO ratio (table 1). Figure 1(a2-e2) shows the SEM images of PHBV porous fibers after incubating in SBF solution for 4 weeks, from which we can see that the obtained porous fiber diameter also increased with the increase in PEO ratio. Meanwhile, the diameters of PHBV porous fibers also increased when compared to corresponding as-spun fibers. The diameter of each type of PHBV fibers (as-spun fibers and porous fiber with different PEO ratio) are shown in table 1.

3.1.2. Pore percentage, size and distribution. From figure 1(a2-e2) it can be found that pores appeared on the surface of PHBV fibers after immersion in SBF solution for 4 weeks when PEO ratio reached $30 \%$. Figure 2(a) shows the porosity of porous PHBV fibers, from which it can be seen that porosity of fibers depends on the ratio of PEO. With the increase of PEO ratio in the as-spun fibers, more pores appeared on the surface of PHBV porous fibers. Figure 2(c-d) shows the pore size and distribution of these three types of PHBV porous fibers. It can be seen that with a higher percentage of PEO leaching from PHBV fibers, bigger pores formed (figure 2(c)). From the size distribution results, it was found that less than $30 \%$ of pores are bigger than $100 \mathrm{~nm}$ when PEO's ratio was $30 \%$ and $40 \%$ (figure 2(d), (e)), whereas when PEO reached 50\%, more than $80 \%$ of pores are bigger than $100 \mathrm{~nm}$ (figure 2(f)).

3.1.3. Pore formation in $\mathrm{SBF}$ and $d_{2} \mathrm{O}$. Figure 3 shows the pore formation on PHBV electrospun fibers with $50 \%$ of PEO after immersing in SBF solution and $\mathrm{dH}_{2} \mathrm{O}$ for 4 weeks. It can be seen that 
PHBV fibers immersed in SBF solution formed more pores than those immersed in $\mathrm{dH}_{2} \mathrm{O}$. From the higher magnification image it was found that mineral depositation appeared around the small pores on PHBV electrospun fibers (figure 3(c)).

3.1.4. Residual weight of PHBV fibers. Figure 2(b) shows the residual weight of PHBV fibers after incubation in SBF solution for 2 and 4 weeks. All five kinds of PHBV fibers, irrespective of PEO ratio, lose weight dramatically in the first two weeks- though the weight loss became slower in the subsequent two weeks.

\subsection{Characterization of PHBV/PEO films}

Figure 4((a1)-(e1)) shows the bright field microscope images of PHBV films with different ratios of PEO before incubating in SBF solution. From these images it can be seen that PHBV and PEO formed separate phases. In all five types of films, phase domains of PEO were larger than $3 \mu \mathrm{m}$. Figure 4((a2)-(e2)) shows the images of PHBV films after immersing in SBF solution for 2 weeks. It was shown that large pores formed after PEO removal.

\subsection{Mineralization of fibers and films}

Table 2 shows the percentage of calcium on the surface of PHBV fibers and films after immersing in SBF solution for 4 weeks. From the quantity of elements results we can see that on the surface of fibers, there were relatively higher and consistent minerals than on films with the same ratio of PEO.

\subsection{Cell behavior on fibers}

CCK-8 kit was used to quantitatively analyze the cell proliferation after culture for 1,3 and 5 days on the PHBV as-spun fibers. As shown in figure 5, one day after cell seeding there were no significant 
differences of cell attachment among all groups, which means the changes of diameters and PEO ratio of these as-spun fibers have not profoundly affected initial cell attachment. After 3 and 5 days of culture, cell numbers increased dramatically on as-spun fibers with $10 \%$ and $20 \%$ PEO, while cells grew slowly on as-spun fibers with $30 \%$ and $40 \%$ PEO. When the PEO ratio reached 50\%, cell proliferation was inhibited completely. Figure 5(b) shows the cell proliferation on PHBV as-spun fibers and porous fibers with $40 \%$ PEO, and as-spun fibers with $10 \%$ PEO were chosen as a control. The data demonstrates that cell proliferation on porous fibers was much faster than that on as-spun fibers with $10 \%$ and $40 \%$ PEO.

Figure 6 shows the SEM images of MSCs on the surfaces of PHBV fibrous scaffolds. It shows the same trend with the CCK-8 result. There is the highest cell number on the surface of PHBV porous fibers when compared to PHBV fibers with $10 \%$ and $40 \%$ PEO (figure 6(a, c, e)). From the higher magnification images, it can be seen that after 5 days culture, cells attached and stretched on these three types of fiber surface (figure 6(b, d, f)).

\section{Discussion}

Electrospinning is the most common technology to obtain nanofibers which mimic the extracellular matrix. Typically, the surface of fibers fabricated via electrospinning is smooth. Nanofibers with porous surfaces can present more advantages for a wide variety of applications. In this study, we developed a facile technique to generate multiple pores on electrospun PHBV fibers. The pore size and density can be precisely controlled through the percentage of porogen component, PEO. The pore percentage on PHBV fibers can reach as high as $18 \%$. Interestingly, using SBF to simultaneously remove the PEO component and crystallize calcium phosphate on PHBV fibers enhanced both pore formation and the 
mineral deposition. The pore formation mechanisms on electrospun fibers and cast films were apparently different, leading to a nanometer scale size on fiber and micron scale on film.

PEO is an effective hydro-soluble porogen. It can be removed by aqueous solution or pure water. In this study, the quantity of nanometer sized pores obtained on the surface of PHBV electrospun fibers, as well as their size, increased with an increase in PEO ratio (figure 2). The decrease in mass of PHBV as-spun fibers after submersion in SBF solution for 2 weeks was very close to the mass of PEO pinpointing towards efficient PEO removal. At the same time, we found that the size of pores formed on PHBV/PEO casting films after submersion in SBF solution for 2 weeks was at micron scale, which indicated that in a solvent casting film the PEO domain was much larger than that of electrospun fibers. Tan et al. noted that PHBV and PEO are miscible in the amorphous state, while they form separated crystal structures in the solid state [23]. During the blended PHBV/PEO film forming, the solvent evaporated very slowly, which gave enough time for PHBV and PEO to form matrix-dispersed phase morphologies (figure 7(b)). While the solvent evaporation happens in less than one second, the phase separation cannot finish thoroughly and these separated components in the co-solution are elongated at the same extent during spinning and form a co-continuous fiber structure (figure 7(a)). Zhang et al. used hydro-soluble gelatin as a porogen to obtain porous polycaprolactone (PCL) electrospun fibers and verified the phase separation between gelatin and PCL, by differential scanning calorimetry (DSC) and atomic force microscopy (AFM) [9]. However, because PCL and gelatin are non-miscible, the size of pores on PCL fibers after electrospun-phase separation-leaching treatment were much larger than those observed in current PHBV porous fibers. 
We noticed one intriguing phenomenon that there were more pores on PHBV fibers after incubation in SBF solution than in $\mathrm{dH}_{2} \mathrm{O}$ (figure 3). We speculate that the formation of pores and calcium deposits promoted and supported each other. In the beginning, PEO dissolved into water and pores formed, increasing the surface roughness of fibers and providing a nucleus for calcium deposits [24]. The ions in the SBF solution started depositing around the small pores (figure 3(c)), helping the stabilization of pores. Thus, removal of PEO by SBF instead of water simultaneously generated pores and deposited minerals, which benefited the two processes mutually, leading to a synergetic effect, manifesting as higher porosity.

In our previous studies, we found that in PHBV electrospun systems PEO supplementation can significantly increase the viscosity of the solution, reducing the required concentration of the solution, therefore reducing the obtained PHBV fiber diameter and overall improving their mechanical properties[8]. In this study, PEO was not only supplied as a porogen but also to affect the properties of PHBV/PEO as-spun fibers. According to our previous study, when $10 \%$ PEO was blended, the diameter and continuity of PHBV fibers improved dramatically [8]. Interestingly, the diameter of PHBV as-spun fibers increased slightly when more PEO was blended, probably due to an increase in the viscosity of the solution. Additionally, the increase of PEO ratio also affected the topography of PHBV as-spun fibers. When the ratio of PEO reached 30\%, a striated surface appeared on the surface of blended fibers. Alessandra et al. also observed a similar phenomenon and suggested that these results could be ascribed to a heterogeneous distribution and shrinkage upon solvent evaporation, owing to segregation of the polymeric components into separated domains along the fibers [21]. 
Calcium deposition plays an important role in both bone and tooth formation, as well as pathological conditions, for example atherosclerosis. In bone tissue engineering, polymers composed of ceramic are often employed to construct 3-dimensional scaffolds mimicking the composition of bone. The most frequently used method to construct the polymer/ceramic scaffolds is biomimetic mineralization, in which calcium can deposit onto polymer scaffolds in SBF solution [25]. In order to facilitate calcium deposition in SBF, surface modification is often adopted to introduce functional groups, such as carboxyls, to increase apatite nucleation $[22,26]$. In our study, SBF solution was used to remove the PEO from PHBV as-spun fibers and films. The element analysis results obtained by SEM-EDX indicated there was calcium deposition on the surface of PHBV porous fibers and films. Compared to non-porous PHBV fibers, porous fibers have a slightly higher percentage of calcium, indicating that the nanometer porous topography on the surface of fibers effects calcium deposition. The calcium deposition increased alongside the increase of original PEO percentage on PHBV films, which can be explained by the increasing roughness of PHBV films after PEO dissolving. Because of the high surface area to volume ratio of electrospun fibers, there was a higher calcium percentage on porous fibers than on films. Similarly, Wang et al. also found that the morphology of the surface significantly influenced the apatite-inducing ability [27]. The enhanced mineralization rate on the nanoporous PHBV fibers will compensate for the reduction of mechanical strength due to the presence of nanopores. Further studies are required to optimize the two processes to allow the resultant materials to fit with specific biomedical applications.

As a consequence of the ability to reduce surface protein adsorption, PEO is usually used as a surface modifying additive to prevent platelet adhesion. In the cell culture experiment, our results indicated that 
$50 \%$ blended PEO would inhibit cell proliferation. However, in other conditions, cell proliferation was only slowed down because of the PEO addition. Cell proliferation can also be affected by the topographical properties of scaffolds. In our previous study, we have demonstrated that electrospun fiber diameter and orientation significantly affects cell behavior [8]. In this study, we found that the nanopores on the surface of PHBV fibers can enhance cell proliferation, indicating that MSCs are very sensitive to the environment, especially on the nano scale. It has been reported that nanoscale geometry of $\mathrm{TiO}_{2}$ nanotubes can influence the osteogenic differentiation of MSCs [28]. Although the osteogenic differentiation of MSCs on PHBV porous fibers still warrants further investigation, we can hypothesize that PHBV porous fibers generated by $40 \%$ PEO, possessing high nanometer pore percentage and calcium deposition, would constitute a good candidate for bone tissue engineering.

\section{Conclusion}

In this study, we established a facile technique to generate porous PHBV fibers using PEO as the porogen. The percentage of PEO in the intimal blend polymer (10 to 50\%), the fabrication technique (electrospinning versus cast film) and the removal solvent for PEO (SBF versus water) determined the pore size, number and morphology. Fast solidification of PHBV/PEO blend by electrospinning led to nanometer scale pores on the resulted PHBV fibers; whilst slow processing for films resulted in micron size pores due to incomplete phase separation. Simultaneously, removal of PEO and deposition of calcium by SBF enhanced pore formation in terms of pore percentage. As a result, porous fibers with nanometer scale pores can induce and accelerate calcium deposition. The porous and mineralized surface of fibers exhibited excellent features to promote MSC proliferation. 


\section{Acknowledgements}

This work was supported by the National Science Foundation of China (No. 31340049) and EC FP7 Marie Curie International Incoming Fellowship. 


\section{References}

[1] Zhang JJ, Liu J, Yu H, Zhang Y, Zhu MF, Chen YM. Crosslinked Electrospun UPM/PHBV/PVP Fibers for Sustained Drug Release. Mater Sci Forum 2009;610-613:1331-4.

[2] Lu LX, Zhang XF, Wang YY, Ortiz L, Mao X, Jiang ZL, et al. Effects of hydroxyapatite-containing composite nanofibers on osteogenesis of mesenchymal stem cells in vitro and bone regeneration in vivo. ACS applied materials \& interfaces 2013;5:319-30.

[3] Biazar E, Keshel SH. Gelatin-Modified Nanofibrous PHBV Tube as Artificial Nerve Graft for Rat Sciatic Nerve Regeneration. International Journal of Polymeric Materials 2014;63:330-6.

[4] Kuppan P, Vasanthan KS, Sundaramurthi D, Krishnan UM, Sethuraman S. Development of poly(3-hydroxybutyrate-co-3-hydroxyvalerate) fibers for skin tissue engineering: effects of topography, mechanical, and chemical stimuli. Biomacromolecules 2011;12:3156-65.

[5] Wang YY, Lu LX, Shi JC, Wang HF, Xiao ZD, Huang NP. Introducing RGD peptides on PHBV films through PEG-containing cross-linkers to improve the biocompatibility. Biomacromolecules 2011;12:551-9.

[6] Jack KS, Velayudhan S, Luckman P, Trau M, Grondahl L, Cooper-White J. The fabrication and characterization of biodegradable HA/PHBV nanoparticle-polymer composite scaffolds. Acta biomaterialia 2009;5:2657-67.

[7] Sultana N, Wang M. Fabrication of HA/PHBV composite scaffolds through the emulsion freezing/freeze-drying process and characterisation of the scaffolds. J Mater Sci-Mater M 2008;19:2555-61.

[8] Lu LX, Wang YY, Mao X, Xiao ZD, Huang NP. The effects of PHBV electrospun fibers with different diameters and orientations on growth behavior of bone-marrow-derived mesenchymal stem cells. Biomedical materials 2012;7:015002.

[9] Zhang YZ, Feng Y, Huang ZM, Ramakrishna S, Lim CT. Fabrication of porous electrospun nanofibres. Nanotechnology 2006;17:901-8. 
[10] Greiner A, Wendorff JH. Electrospinning: a fascinating method for the preparation of ultrathin fibers. Angewandte Chemie 2007;46:5670-703.

[11] Pham QP, Sharma U, Mikos AG. Electrospinning of polymeric nanofibers for tissue engineering applications: a review. Tissue engineering 2006;12:1197-211.

[12] Barhate RS, Ramakrishna S. Nanofibrous filtering media: Filtration problems and solutions from tiny materials. Journal of Membrane Science 2007;296:1-8.

[13] Greiner A, Wendorff JH. Electrospinning: A fascinating method for the preparation of ultrathin fibres. Angew Chem Int Edit 2007;46:5670-703.

[14] Bognitzki M, Czado W, Frese T, Schaper A, Hellwig M, Steinhart M, et al. Nanostructured fibers via electrospinning. Adv Mater 2001;13:70-2.

[15] Casper CL, Stephens JS, Tassi NG, Chase DB, Rabolt JF. Controlling surface morphology of electrospun polystyrene fibers: Effect of humidity and molecular weight in the electrospinning process. Macromolecules 2004;37:573-8.

[16] McCann JT, Marquez M, Xia YN. Highly porous fibers by electrospinning into a cryogenic liquid. J Am Chem Soc 2006;128:1436-7.

[17] Mehraban M, Zadhoush A, Ravandi SAH, Bagheri R, Tehrani AH. Preparation of Porous Nanofibers from Electrospun Polyacrylonitrile/Calcium Carbonate Composite Nanofibers Using Porogen Leaching Technique. Journal of Applied Polymer Science 2013;128:926-33.

[18] Cacciotti I, Calderone M, Bianco A. Tailoring the properties of electrospun PHBV mats: Co-solution blending and selective removal of PEO. Eur Polym J 2013;49:3210-22.

[19] Nasir M, Matsumoto H, Minagawa M, Tanioka A, Danno T, Horibe H. Preparation of Porous PVDF Nanofiber from PVDF/PVP Blend by Electrospray Deposition. Polymer Journal 2007;39:1060-4. 
[20] Li X, Liu KL, Wang M, Wong SY, Tjiu WC, Bin He C, et al. Improving hydrophilicity, mechanical properties and biocompatibility of poly[(R)-3-hydroxybutyrate-co-(R)-3-hydroxyvalerate] through blending with poly[(R)-3-hydroxybutyrate]-alt-poly(ethylene oxide). Acta biomaterialia 2009;5:2002-12.

[21] Bianco A, Calderone M, Cacciotti I. Electrospun PHBV/PEO co-solution blends: Microstructure, thermal and mechanical properties. Mat Sci Eng C-Mater 2013;33:1067-77.

[22] Oyane A, Uchida M, Choong C, Triffitt J, Jones J, Ito A. Simple surface modification of poly(epsilon-caprolactone) for apatite deposition from simulated body fluid. Biomaterials 2005;26:2407-13.

[23] Tan SM, Ismail J, Kummerlöwe C, Kammer HW. Crystallization and melting behavior of blends comprising poly(3-hydroxy butyrate-co-3-hydroxy valerate) and poly(ethylene oxide). Journal of Applied Polymer Science 2006;101:2776-83.

[24] Dey A, Bomans PH, Muller FA, Will J, Frederik PM, de With G, et al. The role of prenucleation clusters in surface-induced calcium phosphate crystallization. Nature materials 2010;9:1010-4.

[25] Chen J, Chu B, Hsiao BS. Mineralization of hydroxyapatite in electrospun nanofibrous poly(L-lactic acid) scaffolds. Journal of biomedical materials research Part A 2006;79:307-17.

[26] Yang F, Wolke JGC, Jansen JA. Biomimetic calcium phosphate coating on electrospun poly( $\varepsilon$-caprolactone) scaffolds for bone tissue engineering. Chemical Engineering Journal 2008;137:154-61.

[27] Wang XJ, Li YC, Lin JG, Yamada Y, Hodgson PD, Wen CE. In vitro bioactivity evaluation of titanium and niobium metals with different surface morphologies. Acta biomaterialia 2008;4:1530-5.

[28] Lv L, Liu Y, Zhang P, Zhang X, Liu J, Chen T, et al. The nanoscale geometry of TiO2 nanotubes influences the osteogenic differentiation of human adipose-derived stem cells by modulating $\mathrm{H} 3 \mathrm{~K} 4$ trimethylation. Biomaterials 2015;39:193-205. 
Table 1 Diameter of PHBV as-spun fibers and porous fibers

\begin{tabular}{ccc}
\hline & As-spun fibers $(\mathrm{nm})$ & Porous fibers $(\mathrm{nm})$ \\
\hline PHBV:PEO=9:1 & $623 \pm 48$ & $811 \pm 59$ \\
PHBV:PEO=8:2 & $827 \pm 68$ & $938 \pm 85$ \\
PHBV:PEO=7:3 & $898 \pm 84$ & $1230 \pm 129$ \\
PHBV:PEO=6:4 & $919 \pm 125$ & $1272 \pm 153$ \\
PHBV:PEO=5:5 & $1164 \pm 126$ & $1293 \pm 160$ \\
\hline
\end{tabular}

Table 2 Calcium percentage on porous fibers and films. $(*): P<0.05$

\begin{tabular}{|c|c|c|c|c|c|}
\hline & $: 5(\%)$ & :4 (\%) & 7:3(\%) & $3: 2(\%)$ & )$: 1(\%)$ \\
\hline \multirow{3}{*}{ m-4 weeks } & $1.38 \pm$ & & & ) $.075 \pm$ & 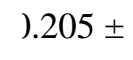 \\
\hline & & $1 \pm 0.02$ & $25 \pm 0.15$ & & \\
\hline & 0.07 & & & 0.004 & 0.015 \\
\hline \multirow[t]{2}{*}{ Fibers-4 } & & $.51 \pm$ & $.335 \pm$ & ) $.425 \pm$ & $0.335 \pm$ \\
\hline & $1 \pm 0.09$ & & & & \\
\hline weeks & & $0.07 *$ & 0.035 & 0.135 & 0.035 \\
\hline
\end{tabular}




\section{Figure Captions}

Figure 1 SEM images of PHBV as-spun fibers and porous fibers with a PEO percentage of 10\% (a1, a2), 20\% (b1, b2), 30\% (c1, c2), 40\% (d1, d2), and 50\% (e1, e2).

Figure 2 (a) Pore percentage of PHBV porous fibers after PEO being dissolved in SBF; (b) Residual weight of PHBV fibers with different ratios of PEO after immersion in SBF for 2 and 4 weeks; (c) Pore size in fibers of PHBV:PEO = 7:3, 6:4, 5:5; (d-f) Distribution of pore size in PHBV fibers with the ratio of PEO 30\% (d), $40 \%$ (e), and 50\% (f). (*): $P<0.05$

Figure 3 SEM images of PHBV/PEO (5:5) electrospun fibers after immersion in $\mathrm{dH}_{2} \mathrm{O}$ and $\mathrm{SBF}$ for 4 weeks.

(a) PHBV fibers immersed in SBF; (b) PHBV fibers immersed in $\mathrm{dH}_{2} \mathrm{O}$; (c) High magnification images of PHBV fibers immersed in SBF; (d) High magnification images of PHBV fibers immersed in $\mathrm{dH}_{2} \mathrm{O}$.

Figure 4 Bright field images of PHBV films before (a1-e1) and after (a2-e2) immersion in SBF for 4 weeks with different ratios of PEO. PHBV:PEO = (a1, a2) 9:1, (b1, b2) 8:2, (c1, c2) 7:3, (d1, d2) 6:4, and (e1, e2) 5:5.

Figure 5 Viability of MSCs on different PHBV fibers. (a) PHBV as-spun fibers with PEO ratios of 10\%, 20\%, 30\%, 40\%, and 50\%; (b) PHBV as-spun fibers with PEO ratios of $10 \%$ and 40\%, and PHBV porous fibers with a PEO ratio of $40 \%{ }^{a}$ : PHBV fibers with $40 \%$ PEO immersed in SBF solution for 4 weeks. Six parallel repeats were tested for each sample; the bar indicates standard deviation. (\#): $P>0.05,(*): P<0.05,(* *): P<0.01,(* * *): P<0.001$.

Figure 6 SEM images of MSCs after 5 days of culture on ((a)-(b)) PHBV as-spun fibers with 10\% PEO, ((c)-(d)) PHBV porous fibers with 40\% PEO leaching out in SBF, and ((e)-(f)) PHBV as-spun fibers with $40 \%$ PEO. 
Figure 7 Schematic diagram of phase separation in PHBV/PEO (a) electrospun fibers and (b) films. 